\title{
¿Hipoacusia súbita?
}

\section{Sudden Hearing Loss?}

Santiago Cornejo Sch¹, Raimundo García M², José San Martín P¹.

\section{RESUMEN}

Se presenta un caso de paciente mujer sin antecedentes mórbidos que consulta a nuestro servicio por síntomas sugerentes de hipoacusia súbita. La evaluación audiométrica y prueba calórica era compatible con una parálisis cocleovestibular y dado que no presentaba contraindicaciones, se decide iniciar tratamiento con corticoides y antiviral oral. La evolución inicial fue favorable sin embargo al control del mes presentó un nuevo deterioro audiométrico. Esto motivó mayor estudio con el cual se pudo llegar a un diagnóstico etiológico. Este caso es presentado para recordar que hay otras patologías que pueden simular una hipoacusia súbita idiopática, lo cual no se debe olvidar en el manejo de estos pacientes.

Palabras clave: Hipoacusia súbita.

\section{ABSTRACT}

Clinical case of female patient without morbid background who consults due to sudden hearing loss symptoms. The audiometric and caloric tests were compatible with a cochleovestibular paralysis and because there were no contraindications, oral steroids and antiviral treatment was indicated. Although the initial response was favorable, at the first month check up an audiometric deterioration was found. This led to further study achieving an etiological diagnosis. This case is presented to remind that there are other pathologies that can simulate an idiopathic sudden hearing loss, which should not be forgotten in the management of these patients.

Key words: Sudden hearing loss.

\section{INTRODUCCIÓN}

La hipoacusia neurosensorial súbita (HNS) es una patología infrecuente con una incidencia estimada de 2 a 20 por 100.000 habitantes $^{1}$ pero se estima que está subdiagnosticada por la falta de consultas de aquellos pacientes que presentan resolución espontánea². Es definida como la pérdida auditiva sensorioneural idiopática de al menos $30 \mathrm{~dB}$ en 30 más frecuencias consecutivas en un período menor a 3 días $^{3}$. Puede ocurrir a cualquier edad, pero afecta más frecuentemente a la pobla- ción en la quinta década de vida sin observarse diferencia por género ${ }^{4}$. La presentación más frecuente es la unilateral, pero hasta el $3 \%$ de los casos puede ser bilateral' ${ }^{1}$.

La etiopatogenia de la HNS es incierta siendo identificada en menos del $20 \%$ de los casos. El $1 \%$ puede ser la presentación de una patología "retrococlear" como el schwannoma vestibular, la enfermedad desmielinizante y el accidente vascular $^{5}$; entre el $10 \%-15 \%$ se puede identificar otra causa tal como hidrops, trauma, sífilis, fístula perilinfática, etc ${ }^{6}$. Es considerada, por lo tanto, en

1 Médico. Departamento de Otorrinolaringología, Hospital Clínico de la Pontificia Universidad Católica de Chile.

2 Médico, Pontificia Universidad Católica de Chile. 
la mayoría de los casos como idiopática y actualmente se proponen varias teorías etiológicas: vascular, viral, autoinmune, ruptura de membranas y respuesta inflamatoria anormal.

En el último tiempo se ha desarrollado la teoría de una respuesta anormal de la cascada inflamatoria en la cóclea debido a algún estímulo indefinido, que sería compatible con los hallazgos anatomopatológicos, pero se requiere mayor investigación para confirmarlo?.

El pronóstico es variable, dependiendo principalmente de la intensidad de la pérdida auditiva inicial y de la edad del afectado: mientras mayor sea la pérdida inicial y la edad, peor pronóstico auditivo ${ }^{4}$.

Según algunos autores, la recomendación actual es realizar una resonancia magnética (RM) con gadolinio para descartar patología estructural ${ }^{4}$.

\section{CASO CLÍNICO}

Mujer de 47 años sin antecedentes mórbidos. Acude al Departamento de Otorrinolaringología de la Universidad Católica por síntomas iniciados 10 días previo a la consulta caracterizados por hipoacusia de comienzo súbito en oído izquierdo asociado a tinnitus y desequilibrio leve. Al examen físico destacaba nistagmus espontáneo grado 1 y alteración leve de la marcha. El resto del examen otorrinolaringológico era normal.

Se solicitó examen de VIII par que mostraba en el oído izquierdo anacusia y arreflexia vestibular. (audiometría en Figura 1). Además se solicitó una resonancia magnética $(\mathrm{RM})$ de cerebro pero la paciente no se realizó este examen.

Se decide manejar con prednisona en dosis de $1 \mathrm{mg} / \mathrm{kg} /$ día y aciclovir, ambos por 7 días. Con esto cedió el desequilibrio, el tinnitus y mejoró significativamente la audición.

En VIII par controlado a la semana, la audición si bien no llegó a niveles normales, mostró una gran mejoría. El promedio de tonos puros (PTP) aéreo/óseo en el oído izquierdo fue 43,5/37,5 y en el oído derecho 7,5/7,5 (Figura 2). La discriminación mejoró de $0 \%$ a $72 \%$ en el oído izquierdo manteniéndose en $100 \%$ en el oído derecho. En la prueba calórica la arreflexia vestibular izquierda se mantuvo sin cambios.

Por la buena evolución pero sin llegar a niveles normales se decide mantener la prednisona en la misma dosis por 7 días más (14 días en total) y control con audiometría a la semana.
Al volver la paciente a la semana ya estaba totalmente asintomática. La hipoacusia leve que refería en control previo había cedido. La audiometría mostraba una audición más baja en oído izquierdo que en derecho pero ambas dentro de límites normales. El PTP en oído izquierdo era $20 / 20 \mathrm{~dB}$. y en el oído derecho era de 3.8/3,8 dB. (Figura 3). La discriminación subió de $72 \%$ a $88 \%$ en el oído izquierdo.

Por la buena evolución se le indicó suspender gradualmente la prednisona y volver en 2 semanas con nueva audiometría.

Al volver refiere nuevamente hipoacusia en oído izquierdo lo cual es concordante con una nueva audiometría que presentaba caída en la audición. El PTP había empeorado a 48,8/46,3 dB. y la discriminación había caído a 0\% en el oído izquierdo (Figura 4).

Se insistió en la necesidad de contar con una RM de cerebro con gadolinio como medio de

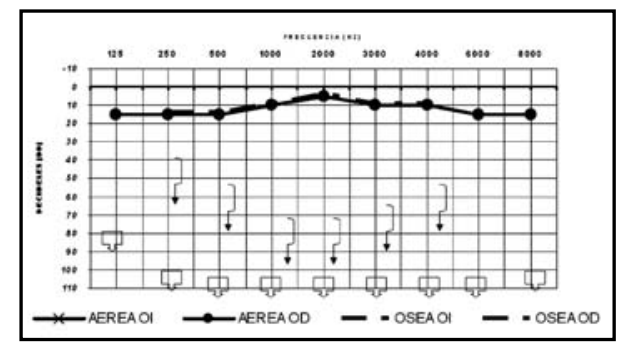

Figura 1. Audiometría inicial.

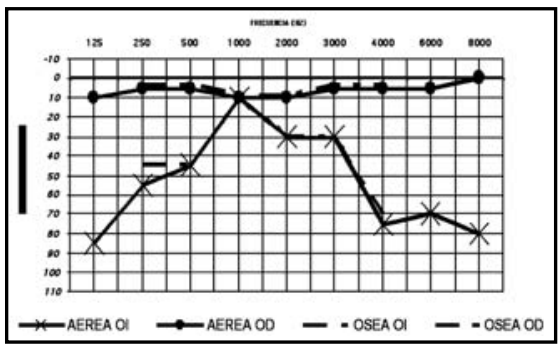

Figura 2. Audiometría de control a los 7 días de tratamiento con prednisona y aciclovir.

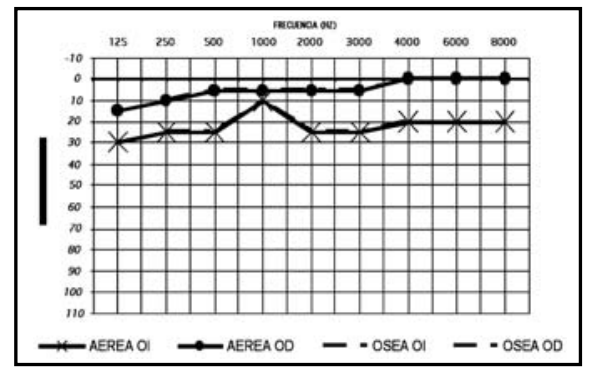

Figura 3. Audiometría de control a los 14 días de tratamiento esteroidal. 


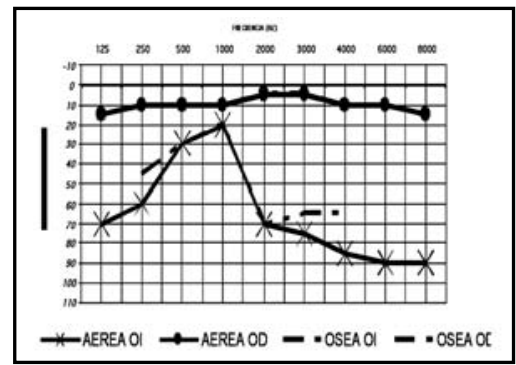

Figura 4. Audiometría de control a los 28 días de iniciado el tratamiento.

contraste, encontrando un Schwannoma vestibular izquierdo de $12 \times 9 \mathrm{~mm}$. (Figura 5).

A las 8 semanas se le solicitó nueva audiometría que no mostró cambios respecto a la anterior y prueba calórica mínima que confirmó una parálisis vestibular global. Al momento del diagnóstico del schwannoma vestibular se le informó a la paciente de la patología así como sus alternativas de tratamiento. Luego de interconsultar otros especialistas la paciente opta por la radioterapia. Once meses después del diagnóstico la paciente se sometió a radiocirugía en el Hospital Clínico UC. Diez meses posterior al tratamiento la paciente ha tenido una evolución favorable, no ha presentado síntomas vestibulares y su audición se ha mantenido estable (Figura 6). La motilidad facial es normal y simétrica. El control imagenológico mostró una reducción significativa del tumor (Figura 7).

\section{DISCUSIÓN}

El schwannoma vestibular (SV) es el tumor más frecuente $(80 \%-90 \%)$ del ángulo pontocerebeloso y corresponde al $8 \%$ de los tumores intracraneales en adultos. Es un tumor benigno que se desarrolla a partir de las células de Schwann de la porción vestibular del

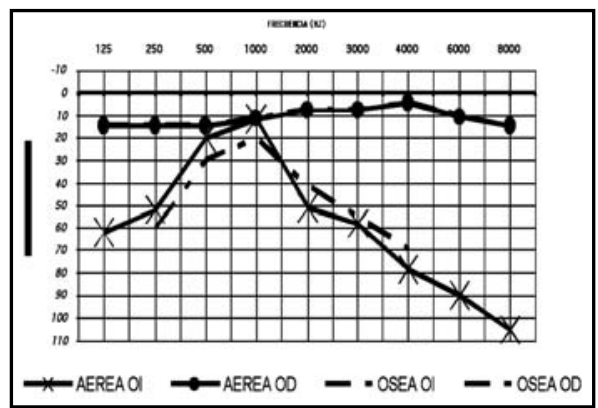

Figura 6 . Audiometría de control posradiocirugía.

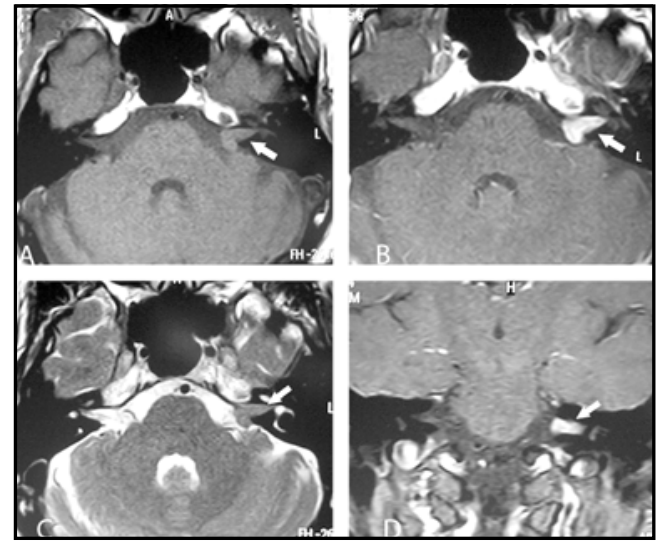

Figura 5. A: Corte axial en T1. B: Corte axial en T1 con gadolinio. C: Corte axial en T2. D: Corte coronal en T1 con gadolinio. Flechas muestran el Schwannoma vestibular.

VIII nervio craneano y su incidencia global es de 1 en 100.000 , pero últimamente se ha notado un incremento de éste, probablemente debido al diagnóstico incidental de lesiones asintomáticas por el mayor número de neuroimágenes realizadas ${ }^{8}$. La edad media de diagnóstico es de 50 años afectando por igual a hombres y mujeres.

La presentación clínica del SV está dada por el compromiso nervioso (trigémino, facial y vestibulococlear), la compresión cerebelosa o la progresión tumoral hacia la fosa posterior. El nervio coclear es el más comprometido (95\%) manifestándose como hipoacusia sensorioneural (HSN) descendente asociada a mala discriminación. El

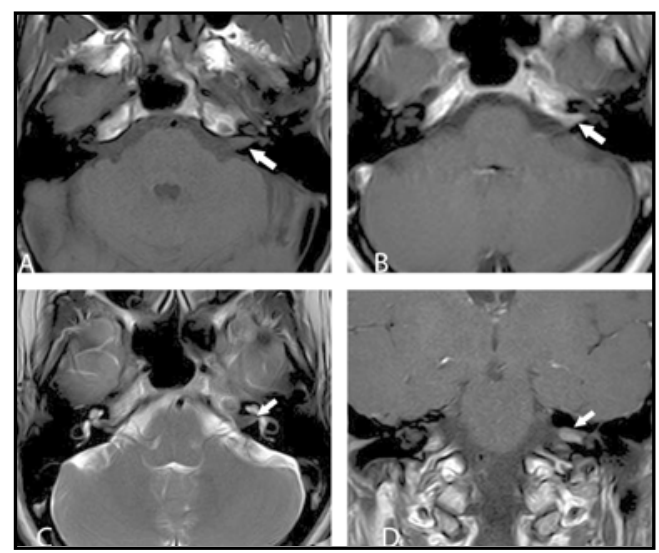

Figura 7. RM muestra que el SV ha reducido de tamaño a expensas de componente cisternal, persistiendo ocupación del conducto auditivo interno. A: Corte axial en T1. B: Corte axial en T1 con gadolinio. C: Corte axial en T2. D: Corte coronal en T1 con gadolinio. Flechas muestran Schwannoma vestibular. 
$63 \%$ de los pacientes presenta además tinnitus. El nervio vestibular se ve afectado en $61 \%$ con alteraciones leves o moderadas del equilibrio, siendo el vértigo poco frecuente. El trigémino y el facial se comprometen en $17 \%$ y $6 \%$, respectivamente 9 .

El SV se presenta más frecuentemente como HSN unilateral 0 asimétrica con 0 sin tinnitus unilateral. Ante la sospecha diagnóstica se debe realizar una RM, el problema radica en que sólo el $2 \%$ de los pacientes con los síntomas clásicos descritos tienen un SV por lo que se ha tratado de establecer criterios para seleccionar mejor a los pacientes y evitar las RM "en blanco" con los costos que esto significa, sin embargo no se ha llegado a consenso ${ }^{10}$. El último intento corresponde a la "regla 3.000" en que los autores concluyen que si existe una diferencia entre ambos oídos mayor a $15 \mathrm{~dB}$ en la frecuencia $3.000 \mathrm{~Hz}$ se debe realizar una RM porque predice con una sensibilidad y especificidad de $73 \%$ y $76 \%$, respectivamente la posibilidad de un SV. En aquellos pacientes con menos de $15 \mathrm{~dB}$ recomiendan el seguimiento con una audiometría bianual debido a que el método falla en diagnosticar aquellos SV de menor tamaño ${ }^{11}$.

En muchos centros de nuestro país existe una alta demanda asistencial, recursos limitados y poco control sobre la población dependiente del sistema. Esto hace difícil mantener un adecuado seguimiento de estos pacientes. Si se sospecha una HNS y se tiene una respuesta inicial favorable, tal como fue este caso, podría ser que el paciente sea dado de alta no llegando al diagnóstico etiológico.

La mejoría sintomática y audiométrica en esta paciente pudo deberse a que los SV también pueden manifestarse como HNS que mejoran en corto plazo, así como al uso de corticoides que pudo llevar a una disminución inicial del edema relacionado con la lesión. Gracias a los controles audiométricos se sospechó una lesión estructural, llegando al diagnóstico al solicitar la RM.

La recomendación actual para algunos autores es realizar una RM a todo paciente con HNS. Esto puede ser difícil en nuestro medio debido al costo elevado de este examen y a que aún no es una herramienta de fácil acceso. Afortunadamente en los últimos años ha habido un aumento en la disponibilidad de este examen, no sólo en la salud privada sino también en los servicios públicos de salud, lo que sin duda ayudará a mejorar el diagnóstico de esta enfermedad, y en consecuencia, su tratamiento y pronóstico.

Creemos que es recomendable que a todo paciente con HNS se le realice controles audiométricos. Estos controles sirven para documentar la recuperación, guiar la rehabilitación y monitorizar recaídas 0 nuevos eventos en ambos oídos que podrían reflejar una patología no diagnosticada inicialmente ${ }^{4}$.

Siempre se debe tener en cuenta que una respuesta inicialmente favorable a la terapia no descarta la necesidad de realizar imágenes.

\section{BIBLIOGRAFÍA}

1. Fetterman BL, Luxford WM, Saunders Je. Sudden bilateral sensorineural hearing loss. Laryngoscope 1996; 106: 1347.

2. SIMmons FB. Sudden idiopathic sensorineural hearing loss: some observations. Laryngoscope 1973; 83: 1221-7.

3. National Institute of Health. Sudden Deafness. Bethesda, MD. National Institutes of Health; 2000. $\mathrm{NIH}$ publication 00-4757.

4. RauCH SD. Clinical practice. Idiopathic sudden sensorineural hearing loss. N Engl J Med 2008; 359: 833.

5. ShaiA FT, SheEHy JL. Sudden sensorineural hearing impairment: a report of 1,220 cases. Laryngoscope 1976; 86: 389-98.

6. Hughes GB, Freedman MA, Haberkamp TJ, Guay ME. Sudden sensorineural hearing loss. Otolaryngol Clin North Am 1996; 29: 393-405.

7. Merchant SN, Durando ML, Adams JC. Sudden Deafness: Is It Viral? ORL J Otorhinolaryngol Relat Spec 2008; 70(1): 52-62.

8. Lin D, Hegarty JL, Fischbein NJ, Jackler RK. The prevalence of 'incidental' acoustic neuroma. Arch Otoloryngol Head Neck Surg 2005; 131: 241-4.

9. Matriles C, Samu M. Management of 1000 vestibular schwannomas (acoustic neuromas): clinical presentation. Neurosurgery 1997; 40: 1-9.

10. Cueva RA. Auditory brainstem response versus magnetic resonante imaging for the evaluation of asymmetric sensorineural hearing loss. Laryngoscope 2004; 114: 1686-92.

11. Saliba I, Martineau G, Chagnon M. Asymmetric Hearing Loss: Rule 3,000 for Screening Vestibular Schwannoma. Otol Neurotol 2009; 30: 515-21. 